\title{
Plasma 25-hydroxyvitamin D level and risk of frailty among Chinese community-based oldest-old: evidence from CLHLS study
}

\author{
Qi Xiao \\ Tongji Hospital, Tongji Medical College of Huazhong University of Science and Technology \\ Meiliyang Wu \\ Tongji Hospital, Tongji Medical College of Huazhong University of Science and Technology \\ Jinrui Cui \\ Tongji Hospital, Tongji Medical College of Huazhong University of Science and Technology \\ Mengmei Yuan \\ Tongji Hospital, Tongji Medical College of Huazhong University of Science and Technology \\ Ye Chen \\ Tongji Hospital, Tongji Medical College of Huazhong University of Science and Technology \\ Tieying Zeng ( $\triangle 984451641 @ q q . c o m)$
}

\section{Research article}

Keywords: China, oldest-old, frailty, 25(OH)D level, biomarkers

Posted Date: October 14th, 2019

DOI: https://doi.org/10.21203/rs.2.15995/v1

License: (a) This work is licensed under a Creative Commons Attribution 4.0 International License. Read Full License

Version of Record: A version of this preprint was published at BMC Geriatrics on April 6th, 2020. See the published version at https://doi.org/10.1186/s12877-020-01523-w. 


\section{Abstract}

Background: Whether vitamin D deficiency may contribute to frailty remains inconclusive in Chinese older adults. The aim of this study was to examine the association between 25-hydroxyvitamin D [25(OH)D] level and risk of frailty among Chinese oldest-old in the communities.

Methods: Secondary analysis of data compiled by the 2011 wave of the Chinese Longitudinal Healthy Longevity Survey ( $\mathrm{n}=1324$ ) was extracted. Frailty was assessed by the Study of Osteoporotic Fractures (SOF) index. Multivariate logistic regression with spine smoothing was performed to investigate the association between 25(OH)D level and risk of frailty after adjusting for socio - demographic variables, health characteristics and confounding biomarkers.

Results: The mean age was $92.89 \pm 7.92$ years, and $844(63.7 \%)$ of participants were women. In all, 426 (33.2\%, 95\% confidence interval, Cl: $29.66-34.69)$ frail participants were recorded. After adjustment for confounding covariates, the level of $25(\mathrm{OH}) \mathrm{D}$ is significantly related to frailty. By the spline smoothing and threshold effect analysis, a monotonically negative association between $25(\mathrm{OH}) \mathrm{D}$ and frailty was identified. Subgroup analyses revealed that the association did not differ by gender and age .

Conclusions: Plasma 25(OH)D was inversely associated with the risk of frailty among Chinese oldest-old. The findings indicate the practical significance of monitoring and managing plasma $25(\mathrm{OH}) \mathrm{D}$ level in the elders.

\section{Background}

With the dramatic population aging, growing attention has been given to the aging-related issues. Frailty is defined as a geriatric syndrome representing a reduced ability to re-build homeostasis in response to external stressors during daily life(1). Frailty in the elderly is well established to be related to long-term adverse health outcomes (such as falls, depression, disability, dependency, and mortality) which were not utterly explained by aging, function decline, or comorbidities (2-5).

There are two main methods of diagnosing and assessing frailty in existing studies: the Frailty Index and Frailty Phenotype (6-9). The Frailty Index (FI) is based on the comprehensive geriatric assessment (CGA) of overall health decline by the accumulation of deficits in multiple domains(10). The Frailty Phenotype(7), by contrast, proposed from the Cardiovascular Health Study (CHS), was focused on the physical aspect of frailty(11). Considering the Frailty Index needs geriatric skills for collecting the information and time-consuming, and the Frailty Phenotype requires the use of specialized equipment, both measurement modalities, however, are less likely to be readily available in clinical settings and therefore have limited utility(12).

The Study of Osteoporotic Fractures (SOF) index(13), as a simplified screening tool of the physical aspect of frailty, was developed from the limited version of the Frailty Phenotype. The SOF index employed only 3 simple self-reported frailty components(12): muscle strengths, low energy, and unintentional weight loss. It has shown an operational definition to the association with falls, disability, fracture, and death(13, 14), and demonstrated the reliability of risk prediction in clinical practice at the population level $(12,15,16)$.

Vitamin D, which primarily synthesized in the skin upon the exposure to sunlight, is necessary for human's musculoskeletal health maintenance(17); its deficiency is proved to be the cause of muscle weakness(18), sarcopenia(19), falls(20), and fracture(21). Evidence has suggested that the low plasma $25(\mathrm{OH})$ D concentration is related to the risk of frailty referring to different ethnic populations(22).

The underlying pathogenic mechanisms could be explained through 3 pathways: the first is the invalidation of regulatory effects of vitamin $D$ on calcium flux, mineral homeostasis and protein anabolism in muscle tissue $(23,24)$; the second is bone metabolic disturbance by secondary hyperparathyroidism(25); the last is the possible property of vitamin D on anti-inflammation(26).

For the present, following limitations with previous literature need to be addressed(27-40):

- The number of oldest-old participants was limited in most studies. Majority of studies were conducted with a minimal number of samples aged more than 80 years.

- Few linear or curvilinear associations were examined by multivariate logistic regression model with spline smoothing analysis, which is an effective method for examining the shapes of the association.

- Most relevant studies focused on Frailty Index or Frailty Phenotype, proofs of association between SOF index as frailty measurement and vitamin D were lacking.

- Most relevant studies were implemented in developed countries (USA, Germany, and the Netherlands), while studies in developing countries were limited, especially among the oldest-old group.

In response to some of the above limitations, this study aimed to examine the association with plasma 25(OH)D level in the risk of frailty among 1324 older adults of the eight longevity areas in the Chinese Longitudinal Healthy Longevity Survey (CLHLS)(41, 42). We used multivariate logistic regression models with spline smoothing in our primary analyses and adjusted for several important covariates, including socio-demographics, health characteristics, and confounding biomarkers.

\section{Methods}




\section{Study design and participants}

Participants with the biomarker sub-study datasets from the $6^{\text {th }}$ (2011) waves of CLHLS were recruited. CLHLS is the first and largest nationwide, community-based, longitudinal prospective cohort survey, concerning older adults in China(43). It provides information on health status, socioeconomic characteristics, lifestyles of the elderly, including a large percent of the oldest population(42). The in-depth study was launched in the eight longevity areas (Laizhou of Shandong Province, Xiayi of Henan Province, Zhongxiang of Hubei Province, Mayang of Hunan Province, Sanshui of Guangdong Province, Yongfu of Guangxi Autonomous Region, Chengmai of Hainan Province, Rudong of Jiangsu Province) (41). During the in-depth study, the Chinese Center for Disease Control and Prevention (CDC) local network medical doctors conducted physical examinations of the participants, and also collected biomarker datasets contain about 30 indicators on routine blood tests, urine tests and blood biochemical tests(41). More detailed descriptions have been previously published elsewhere(44-46).

Initially, a total of 2439 elderly participants were included in the study. We excluded those of younger age (less than $80, n=834,34.2 \%)$ and missing data on SOF index components $(n=281,11.5 \%)$. Finally, we retained 1324 older adults in this study.

\section{Outcome}

We used the SOF index to assess frailty status. Three components were included in the index: underweight (defined as body mass index <18.5), low energy level (indicated by a positive response to the question "Over the last 6 months, have you been limited in activities because of a health problem?", and inability to stand up from a chair without the assistance of arms. As suggested(47), participants with two or more of the three components were defined as frailty.

\section{Exposure}

Fasting venous blood were collected after an overnight fast from all willing participants. Procedures for the collection and shipment of blood samples were described in detail elsewhere(17). 25(OH)Dis the major form of vitamin D in circulation. Plasma 25(OH)Dwas assayed by an enzyme-linked immunoassay using Immunodiagnostic Systems Limited (IDS Ltd, Boldon, UK). The Plasma 25(OH)Dlevel was expressed as nmol/L.

\section{Covariates}

We adjusted for socio-demographic variables, health characteristics and confounding biomarkers in the models. Socio-demographic variables included age, gender (female/male), marital status (married/other), residence (rural/other), education level (no schooling/ $\geq 1$ years of schooling), co-residence [with family member(s) /other].

Health characteristics included lifestyle and chronic diseases. Lifestyle consisted of smoking (yes/no), drinking (yes/no), and regular exercise (yes/no) at present. Chronic diseases included hypertension, diabetes mellitus, heart diseases, cerebrovascular diseases, and respiratory diseases. Hypertension was defined as systolic blood pressure $\geq 140 \mathrm{mmHg}$ and/or diastolic blood pressure $\geq 90 \mathrm{mmHg}(48)$. Diabetes mellitus was diagnosed by fasting plasma glucose $\geq 7.0 \mathrm{mmol} / \mathrm{L}(17,49)$. Other diseases were identified by items of self-reported common diseases diagnosed by the doctor.

Confounding biomarkers were items conducted by blood routine tests, blood biochemistry tests, and urine routine tests. According to the previous relevant studies(22), following biomarkers which largely investigated in relation with frailty were recruited in this study: 1) inflammatory marker: $\mathrm{C}$ reactive protein; 2) immune marker: counts of leukocytes; 3)clinical markers: plasma albumin, total cholesterol, serum creatinine, high-density lipoprotein cholesterol, low-density lipoprotein cholesterol, and hemoglobin; 4) oxidative stress markers: malondialdehyde and superoxide dismutase. All the standard laboratory techniques were performed by the central clinical lab at Capital Medical University in Beijing. For the present analysis, we treated all the confounding biomarkers as continuous variables.

Overall, few data for most confounding variables were missing (1.05\%). For the missing values, we did multiple imputations by chained equations to increase predictive power(50). Distribution of observed data and imputed data are described in Supplementary Table S1 (see Additional file 1). For all the covariates, the distributions of observed and imputed values did not differ substantially.

\section{Statistical Analysis}

Categorical variables were expressed as numbers and percentages, and continuous data were described as mean (SD) or median (IQR). Characteristics among groups were compared by ANOVA, Kruskal-Wallis test or $\chi^{2}$ test. The interquartile range of 25(OH)D level was used to divide the data into four categories. The cutoff points were $26.13,35.89,50.00 \mathrm{nmol} / \mathrm{L}$.

We used multilayer logistic regression model based on the Likelihood Ratio Test (LRT) to determine the association between plasma 25(OH)D concentration and risk of frailty. Box-Tidwell method was used to test the linearity between logit $\mathrm{P}$ and all the continuous variables(51). Therefore, we used continuous terms for all the biomarkers; we also categorized age as subgroups with $80-89,90-99, \geq 100$ years. Data are reported as odds ratios 
(ORs) and 95\% confidence intervals (Cls) in both unadjusted (model 1) and adjusted logistic regression models (model 2, 3, 4). The adjustment was stratified via three layers: (1) we adjusted for socio-demographics (model 2); and (2) we added health characteristics (model 3); (3) we additionally added confounding biomarkers (model 4). The $p$-value of Hosmer-Lemeshow test $>0.05$ indicating reasonable goodness of fit(52).

We used the spline smoothing analysis and threshold effect analysis, which were implemented based on the generalized additive model (GAM)(53) and the piece-wise regression model(54) respectively to examine the actual shapes of the association between level of $25(\mathrm{OH}) \mathrm{D}$ with the risk of frailty. Subgroup analyses and their interactions were tested in the fully adjusted model to explore whether sex and age would confound the association of 25(OH)D level with risk of frailly. Sensitivity analysis was performed in participants with complete variables, and multiple imputations, separately.

Two-tailed $p$-value $<0.05$ were considered the statistical significance in all the analyses. Statistical analyses were conducted by IBM SPSS Statistics Version 22.0, except for the spline smoothing analysis, threshold effect analysis, and multiple imputations were performed by R software Version 3.4 .3 (http://www.R-project.org) and Empower® (www.empowerstats.com).

(Table 1 in the end of this context should be cited here.)

\section{Results}

\section{Sample characteristics}

The Characteristics of participants were compared according to the categories of 25(OH)D level. Full detailed characteristics of all participants are shown in Table 1. The mean \pm SD age of the study population was $92.89 \pm 7.92$ years, and $63.7 \%$ were women $(n=844)$. Participants with frailty were 426 (33.2\%, 95\% Cl: 29.66-34.69). The median 25(OH)D concentration was $35.89 \mathrm{nmol} / \mathrm{L}$ and participants with higher levels $(35.89-50.00,>50.00$ $\mathrm{nmol} / \mathrm{L})$ were significantly younger than those with lower levels $(\leq 26.13,26.13-35.89 \mathrm{nmol} / \mathrm{L})$, more likely to be male, married, with $\geq 1$ year of schooling and do regular exercise.

\section{Association between the level of 25(OH)D and risk of frailty}

As shown in Table 1, accounted for $48.9 \%, 33.8 \%, 28.0 \%$ and $17.9 \%$ of participants in the lowest to highest $25(\mathrm{OH}) \mathrm{D}$ categories reported frailty. There was a significant inverse association between categorical $25(\mathrm{OH})$ Dlevel and risk of frailty in the multivariate logistic regression models. The ORs and $95 \%$ Cls for the association between categories of $25(\mathrm{OH}) \mathrm{D}$ level and frailty were presented in Table 2. After eliminating the interferences of all confounding factors, the ORs of frailty were $3.239(95 \% \mathrm{Cl}$ : $2.113-4.967, \mathrm{p}<0.001)$ for the lowest category $(\leq 26.13 \mathrm{nmol} / \mathrm{L})$ of $25(\mathrm{OH}) \mathrm{D}$ level, 2.341 (95\% Cl: $1.519-3.609, \mathrm{p}<0.001)$ for the second-lowest $(26.13-35.89 \mathrm{nmol} / \mathrm{L})$, and $1.703(95 \% \mathrm{Cl}: 1.088-2.664, \mathrm{p}=0.20)$ for the third-lowest $(30.33-$ $44.46 \mathrm{nmol} / \mathrm{L})$ compared to the highest level subgroup(>50.00 nmol/L), respectively.

Table 2. The associations between serum level of 25(OH)D (nmol/L) and risk of frailty.

\begin{tabular}{|c|c|c|c|c|}
\hline Variables & Model $1^{\mathrm{a}}$ & Model $2^{b}$ & Model $3^{\mathrm{c}}$ & Model $4^{\mathrm{d}}$ \\
\hline \multicolumn{5}{|l|}{ Categories e } \\
\hline$\leq 26.13$ & $4.964(3.332,7.396)^{* * *}$ & $3.472(2.273,5.303)^{* * *}$ & $3.437(2.248,5.255)^{* * *}$ & $3.239(2.113,4.967)^{* * *}$ \\
\hline $26.13-35.89$ & $2.822(1.881,4.234)^{* * *}$ & $2.414(1.571,3.710)^{* * *}$ & $2.420(1.573,3.723)^{* * *}$ & $2.341(1.519,3.609){ }^{* * *}$ \\
\hline $35.89-50.00$ & $1.835(1.204,2.797)^{* *}$ & $1.526(1.102,2.683)^{*}$ & $1.722(1.102,2.692)^{*}$ & $1.703(1.088,2.664)^{*}$ \\
\hline$>50.00$ & reference & reference & reference & reference \\
\hline \multicolumn{5}{|c|}{${ }^{\text {a }}$ Unadjusted model, OR (95\%CI). } \\
\hline \multicolumn{5}{|c|}{ b Adjusted for socio-demographics, OR (95\%CI). } \\
\hline \multicolumn{5}{|c|}{ c Adjusted for socio-demographics and health characteristics, OR (95\%CI). } \\
\hline \multicolumn{5}{|c|}{ d Adjusted for socio-demographics, health characteristics, and confounding biomarkers, OR (95\%CI). } \\
\hline \multicolumn{5}{|c|}{ e $p$-value for Hosmer-Lemeshow test was 0.653 , prediction in accuracy was $74.3 \%$ in the fully adjusted model. } \\
\hline
\end{tabular}

The dose-response relationship between the level of 25(OH)D and the risk of frailty

Consistent with results displayed in Table 2, a monotonically negative curve was observed for the association between plasma 25(OH)D and risk of frailty in the analysis using spline smoothing ( $p$ for trend $<0.001$, Figure 1$)$.. The $p$-value of the log-likelihood ratio test was 0.032 in the unadjusted model, however, 0.317 in the fully adjusted model, which indicated continuous effect in the piece-wise regression model(54) (Table 3).. 


\section{Subgroup analyses}

Figure 1. The dose-response relationship of $25(\mathrm{OH}) \mathrm{D}$ level and risk of frailty in the full adjusted model. Lines = estimated probability of frailty with $25(\mathrm{OH}) \mathrm{D}$, dotted lines $=95 \%$ confidence intervals.

0

Figure 1. The dose-response relationship of $25(\mathrm{OH}) \mathrm{D}$ level and risk of frailty in the full adjusted model. Lines = estimated probability of frailty with $25(\mathrm{OH}) \mathrm{D}$, dotted lines $=95 \%$ confidence intervals.

Subgroup analyses showed the $p$-value for interaction were 0.9753 for gender and 0.1077 for age, which revealed that the association of $25(\mathrm{OH}) \mathrm{D}$ level with frailty did not significantly differ by gender and age after adjusted for socio-demographics, health characteristics, and confounding biomarkers.

(Figure 2)..

Table 3. Threshold effect analysis of 25(OH)D (nmol/L) using the piece-wise regression model

\begin{tabular}{|c|c|c|}
\hline \multirow[t]{2}{*}{ Variables } & Crude $^{a}$ & Adjusted $^{\mathrm{b}}$ \\
\hline & OR (95\%CI) & OR $(95 \% \mathrm{CI})$ \\
\hline Continuous & $0.967(0.960,0.975)^{* * *}$ & $0.975(0.965,0.984)^{* * *}$ \\
\hline \multicolumn{3}{|l|}{ Cutoff } \\
\hline$\leq 33.96$ & $0.948(0.929,0.967)^{* * *}$ & $0.963(0.939,0.988)^{* * *}$ \\
\hline$>33.96$ & $0.978(0.966,0.989)^{* * *}$ & $0.981(0.966,0.996)^{*}$ \\
\hline$p$-value ${ }^{\mathrm{c}}$ & 0.032 & 0.317 \\
\hline \multicolumn{3}{|c|}{ a Crude: no adjustment. } \\
\hline \multicolumn{3}{|c|}{ b Adjusted for socio-demographics, health characteristics and confounding biomarkers. } \\
\hline
\end{tabular}

\section{Sensitivity analysis}

Figure 2. Subgroup analyses for the association between $25(\mathrm{OH}) \mathrm{D}$ and frailty in the fully adjusted model.

0

Figure 2. Subgroup analyses for the association between $25(\mathrm{OH}) \mathrm{D}$ and frailty in the fully adjusted model.

We performed multivariate analysis in participants with complete variables, and multiple imputations, separately. As displayed in Supplementary Table S2 (see Additional file 1), the present findings showed similar results.

\section{Discussion}

In this community-based study, after adjustment for a variety of potential confounding factors, we observed a robust and monotonically negative association of plasma 25(OH)D with frailty measured by the SOF index among a group of oldest-old population in China. After explored this association in different subgroups of participants, the results further supported the association was consistent across gender and age groups.

\section{Comparison with other studies}

The potential role of 25(OH)D level in frailty has rarely been investigated among the oldest-old individuals. As hypothesized, the present findings suggest that plasma $25(\mathrm{OH}) \mathrm{D}$ level is related to frailty after adjustment of numerous confounding. The findings of from our data have been relatively consistent with previous studies involving older adults in the Netherlands(32, 39), Mexican community-dwelling elderly(30), northern Taiwan residents(40) and older participants in Germany(38).

Few studies demonstrated the shapes of the association between $25(\mathrm{OH})$ Dlevel and risk of frailty. In the study of 1606 old men individuals aged $73.8 \pm$ 5.9 years in the USA, a segmented negative curvilinear association between 25(OH)D level and odds of frailty was identified(28). However, a U-shaped curve instead of the segmented-shaped association was found in the study of 6307 old women in the USA (27). For the present study, we identified a robust and monotonically negative association between plasma $25(\mathrm{OH}) \mathrm{D}$ and frailty in Chinese older adults. 
The presence of chronic diseases, lifestyle, and other biomarkers were studied as potential risk factors of frailty $(7,22,31)$. The association of plasma $25(\mathrm{OH}) \mathrm{D}$ with frailty in our study did not substantially change after adding these covariates in the models. This suggests that plasma $25(\mathrm{OH}) \mathrm{D}$ was independently associated with the risk of frailty. However, it remains inconclusive that other possible factors, not included in this study, might contribute to the relationship between $25(\mathrm{OH})$ Dand frailty.

Due to differences in latitudes, seasons, measurements of frailty, adequacy of adjustment for potential confounders and the clinical heterogeneity between races/ethnicities(55), some variations from previous studies were also founded in this study. Firstly, our study reported that the median 25(OH)D level of the elderly is $35.89 \mathrm{nmol} / \mathrm{L}$, which is lower than studies reported in Taiwan(40), Netherlands(32), and German(34). Besides, the prevalence of frailty measured by SOF index is $33.2 \%$, which makes a glancing comparison to $13 \%$ in the Japanese elderly(16) and $17 \%$ in the USA older women(13) by the same screening tool. Since the $25(\mathrm{OH}) \mathrm{D}$ level and frailty status are closely related to aging(22), these differences could also be partly explained by the oversampling of octogenarians, nonagenarians, and centenarians in this study.

Secondly, it has been reported that the association between $25(\mathrm{OH}) \mathrm{D}$ level and frailty was differed by gender(27, 28, 34, 35, 37). A study of participants in Italy identified that Vitamin D insufficiency was associated with frailty in men, but not in women(37). However, conflict results were found in older adults in the USA(31), Spain(36), and Portugal(35). Our study detected that 25(OH)D level was associated with frailty regardless of gender, which is similar to the study in individuals in Germany(34). In addition, our study also indicated that the association did not differ by age subgroups.

\section{Strength and limitations}

The strengths of the current study are the Chinese community-based sample with a well-validated measurement of frailty. This study performed the multivariate logistic regression with spline smoothing to explore the potential linear or curvilinear association between $25(\mathrm{OH}) \mathrm{D}$ level and frailty. To our knowledge, this is the first study that investigated the relationship between $25(\mathrm{OH}) \mathrm{D}$ level and frailty in a nationwide study of Chinese older people. The present study included a large population of Chinese older adults aged 80 years and over, which allowed robust conclusions to be drawn with respect to this group.

This study also has certain limitations. First, a dichotomous outcome measure for frailty has been used in this study, hence the pre-frail status was not taken into account. Second, some of the clinical diseases adjusted as confounding factors were self-reported. For this condition, we adjusted hypertension and diabetes status by clinical data in place of self-reporting so as to eliminate a degree of information bias. Third, this is a descriptive cross-sectional design that does not allow us to evaluate whether change in 25(OH)D level is a cause or consequence of frailty. The longitudinal design should be employed to investigate whether low $25(\mathrm{OH}) \mathrm{D}$ is associated with the onset or progression of frailty in the future.

\section{Implications to practice}

Based on the instructive findings of the present study, it is possible to give some implications for further practice. First, given the application of the SOF index only involves three components and requires no special equipment, it will potentially offer benefits for screening the physical aspect of frailty among the elderly in community settings. Second, the alarming prevalence of frailty presented in our paper emphasized much attention should be paid to those oldest-old living in the community. Third, the results indicate the practical significance of detecting plasma $25(\mathrm{OH}) \mathrm{D}$ level in older adults. Fourth, it is possible that efforts should be made to investigating whether management of vitamin D deficiency may relieve or prevent the development of frailty in older adults.

\section{Conclusion}

Using the methodology of multivariate logistic regression with spline smoothing, this study used the SOF Index in Chinese community-dwelling population to draw a monotonically negative association between $25(\mathrm{OH}) \mathrm{D}$ level and frailty. It indicates that $25(\mathrm{OH}) \mathrm{D}$ level is identified as a potential biomarker of frailty and targets for intervention, therefore beneficial to gerontological research and practice in frailty.

\section{List Of Abbreviations}

CLHLS, Chinese Longitudinal Healthy Longevity Survey; 25-hydroxyvitamin D, 25(OH)D; SOF, Study of Osteoporotic Fractures; FI, Frailty Index; CGA, comprehensive geriatric assessment; CHS, Cardiovascular Health Study; CDC, Center for Disease Control and Prevention; SD, standard variance; IQR, interquartile range; LRT, Likelihood Ratio Test; OR, odds ratio; Cl, confidence interval; GAM, generalized additive model; CRP, C reactive protein; ALB, plasma albumin; $\mathrm{CHO}$, total cholesterol; CREA, plasma creatine; SOD, superoxide dismutase; HDLC, high-density lipoprotein cholesterol; LDLC, low-density lipoprotein cholesterol; TG, triglyceride; SOD, superoxide dismutase; MDA, malondialdehyde; WBC, white blood cell count; HGB, hemoglobin.

\section{Declarations}

\section{Ethics approval and consent to participate}


The CLHLS study was approved by the Research Ethics Committee of Peking University (IRB00001052-13074), and all participants or their proxy respondents provided written informed consent.

\section{Consent for publication}

Not applicable.

\section{Availability of data and material}

The CLHLS questionnaires are available at https://sites.duke.edu/centerforaging/ programs/chinese-longitudinal-healthy-longevity-survey-clhls/surveydocumentation/ questionnaires/. The full datasets used in this analysis are available from the corresponding author upon reasonable request.

\section{Competing interests}

The authors declare that they have no competing interests.

\section{Funding}

Data of this study is from the National Basic Research Program of China (2013CB530700), National Natural Science Foundation of China (71233001), United States Department of Health and Human Services. National Institutes of Health. National Institute on Aging (R01 AG023627). This study was also supported by the National Natural Science Foundation of China (71573097).

\section{References}

1.Clegg A, Young J, lliffe S, Rikkert MO, Rockwood K. Frailty in elderly people. The Lancet. 2013;381(9868):752-62.

2.Collard RM, Boter H, Schoevers RA, Oude Voshaar RC. Prevalence of Frailty in Community-Dwelling Older Persons: A Systematic Review. Journal of the American Geriatrics Society. 2012;60(8):1487-92.

3.Kojima G. Frailty as a Predictor of Future Falls Among Community-Dwelling Older People: A Systematic Review and Meta-Analysis. J Am Med Dir Assoc. 2015;16(12):1027-33.

4.Kojima G. Frailty as a predictor of disabilities among community-dwelling older people: a systematic review and meta-analysis. Disabil Rehabil. 2017;39(19):1897-908.

5.At J, Bryce R, Prina M, Acosta D, Ferri CP, Guerra M, et al. Frailty and the prediction of dependence and mortality in low- and middle-income countries: a 10/66 population-based cohort study. BMC Med. 2015;13(1):138.

6.Rockwood K, Mitnitski A. Frailty in Relation to the Accumulation of Deficits. The Journals of Gerontology: Series A. 2007;62(7):722-7.

7.Fried LP, Tangen CM, Walston J, Newman AB, Hirsch C, Gottdiener J, et al. Frailty in older adults: evidence for a phenotype. The journals of gerontology Series A, Biological sciences and medical sciences. 2001;56(3):M146-56.

8.Morley JE, Vellas B, Abellan van Kan G, Anker SD, Bauer JM, Bernabei R, et al. Frailty Consensus: A Call to Action. J Am Med Dir Assoc. 2013;14(6):392-7.

9.Mitnitski AB, Mogilner AJ, Rockwood K. Accumulation of Deficits as a Proxy Measure of Aging. TheScientificWorldJOURNAL. $2001 ; 1$.

10.Rockwood K, Mitnitski A. Frailty Defined by Deficit Accumulation and Geriatric Medicine Defined by Frailty. Clinics in geriatric medicine. $2011 ; 27(1): 17-26$.

11.Cesari M, Gambassi G, Abellan van Kan G, Vellas B. The frailty phenotype and the frailty index: different instruments for different purposes. Age Ageing. 2013;43(1):10-2.

12.Erusalimsky JD, Grillari J, Grune T, Jansen-Duerr P, Lippi G, Sinclair AJ, et al. In Search of 'Omics'-Based Biomarkers to Predict Risk of Frailty and Its Consequences in Older Individuals: The FRAILOMIC Initiative. Gerontology. 2016;62(2):182-90.

13.Ensrud KE, Ewing SK, Taylor BC, Fink HA, Cawthon PM, Stone KL, et al. Comparison of 2 frailty indexes for prediction of falls, disability, fractures, and death in older women. Archives Of Internal Medicine. 2008;168(4):382-9. 
14.Bradlee ML, Mustafa J, Singer MR, Moore LL. High-Protein Foods and Physical Activity Protect Against Age-Related Muscle Loss and Functional Decline. The journals of gerontology Series A, Biological sciences and medical sciences. 2017;73(1):88-94.

15.Buta BJ, Walston JD, Godino JG, Park M, Kalyani RR, Xue Q-L, et al. Frailty assessment instruments: Systematic characterization of the uses and contexts of highly-cited instruments. Ageing Res Rev. 2016;26:53-61.

16.Cable N, Hiyoshi A, Kondo N, Aida J, Sjoqvist H, Kondo K. Identifying Frail-Related Biomarkers among Community-Dwelling Older Adults in Japan: A Research Example from the Japanese Gerontological Evaluation Study. Biomed Res Int. 2018:8.

17.Matchar DB, Chei CL, Yin ZX, Koh V, Chakraborty B, Shi XM, et al. Vitamin D Levels and the Risk of Cognitive Decline in Chinese Elderly People: the Chinese Longitudinal Healthy Longevity Survey. The journals of gerontology Series A, Biological sciences and medical sciences. 2016;71(10):1363-8.

18.Bischoff-Ferrari HA, Dietrich T, Orav EJ, Hu FB, Zhang Y, Karlson EW, et al. Higher 25-hydroxyvitamin D concentrations are associated with better lower-extremity function in both active and inactive persons aged $>$ or $=60 \mathrm{y}$. The American journal of clinical nutrition. 2004;80(3):752-8.

19.Visser M, Deeg DJ, Lips P. Low vitamin D and high parathyroid hormone levels as determinants of loss of muscle strength and muscle mass (sarcopenia): the Longitudinal Aging Study Amsterdam. The Journal of clinical endocrinology and metabolism. 2003;88(12):5766-72.

20.Murad MH, Elamin KB, Abu Elnour NO, Elamin MB, Alkatib AA, Fatourechi MM, et al. Clinical review: The effect of vitamin D on falls: a systematic review and meta-analysis. The Journal of clinical endocrinology and metabolism. 2011;96(10):2997-3006.

21.Hill TR, Aspray TJ. The role of vitamin D in maintaining bone health in older people. Therapeutic advances in musculoskeletal disease. 2017;9(4):8995 .

22.Kane AE, Sinclair DA. Frailty biomarkers in humans and rodents: Current approaches and future advances. Mechanisms of Ageing and Development. 2019;180:117-28.

23.Anagnostis P, Dimopoulou C, Karras S, Lambrinoudaki I, Goulis DG. Sarcopenia in post-menopausal women: Is there any role for vitamin D? Maturitas. 2015;82(1):56-64.

24.Sanders KM, Scott D, Ebeling PR. Vitamin D Deficiency and its Role in Muscle-Bone Interactions in the Elderly. Curr Osteoporos Rep. 2014;12(1):7481.

25.Bruyere O, Cavalier E, Buckinx F, Reginster JY. Relevance of vitamin D in the pathogenesis and therapy of frailty. Current opinion in clinical nutrition and metabolic care. 2017;20(1):26-9.

26.Arnson Y, Amital H, Shoenfeld Y. Vitamin D and autoimmunity: New aetiological and therapeutic considerations. Ann Rheum Dis. 2007;66(9):113742.

27.Ensrud KE, Ewing SK, Fredman L, Hochberg MC, Cauley JA, Hillier TA, et al. Circulating 25-hydroxyvitamin D levels and frailty status in older women. The Journal of clinical endocrinology and metabolism. 2010;95(12):5266-73.

28.Ensrud KE, Blackwell TL, Cauley JA, Cummings SR, Barrett-Connor E, Dam TT, et al. Circulating 25-hydroxyvitamin D levels and frailty in older men: the osteoporotic fractures in men study. Journal of the American Geriatrics Society. 2011;59(1):101-6.

29.Wong YY, McCaul KA, Yeap BB, Hankey GJ, Flicker L. Low vitamin D status is an independent predictor of increased frailty and all-cause mortality in older men: the Health in Men Study. The Journal of clinical endocrinology and metabolism. 2013;98(9):3821-8.

30.Gutierrez-Robledo LM, Avila-Funes JA, Amieva H, Meillon C, Acosta JL, Navarrete-Reyes AP, et al. Association of low serum 25-hydroxyvitamin D levels with the frailty syndrome in Mexican community-dwelling elderly. The aging male: the official journal of the International Society for the Study of the Aging Male. 2016;19(1):58-63.

31.Buta B, Choudhury PP, Xue QL, Chaves P, Bandeen-Roche K, Shardell M, et al. The Association of Vitamin D Deficiency and Incident Frailty in Older Women: The Role of Cardiometabolic Diseases. Journal of the American Geriatrics Society. 2017;65(3):619-24.

32.Vaes AMM, Brouwer-Brolsma EM, Toussaint N, de Regt M, Tieland M, van Loon LJC, et al. The association between 25-hydroxyvitamin D concentration, physical performance and frailty status in older adults. European journal of nutrition. 2019;58(3):1173-81.

33.van den Berg KS, Arts MHL, Collard RM. Vitamin D deficiency and course of frailty in a depressed older population. 2018:1-7.

34.Spira D, Buchmann N, Konig M, Rosada A, Steinhagen-Thiessen E, Demuth I, et al. Sex-specific differences in the association of vitamin D with low lean mass and frailty: Results from the Berlin Aging Study II. Nutrition (Burbank, Los Angeles County, Calif). 2019;62:1-6.

35.Sousa-Santos AR, Afonso C, Santos A. The association between 25(OH)D levels, frailty status and obesity indices in older adults.

2018;13(8):e0198650. 
36.Alvarez-Ríos Al, Guerrero JM, García-García FJ, Rodríguez-Mañas L, Medrano-Campillo P, de la Torre Lanza MA, et al. Associations between frailty and serum N-terminal propeptide of type I procollagen and 25-hydroxyvitamin D in older Spanish women: The Toledo Study for Healthy Aging. Experimental gerontology. 2015;69:79-84.

37.Shardell M, Hicks GE, Miller RR, Kritchevsky S, Andersen D, Bandinelli S, et al. Association of Low Vitamin D Levels With the Frailty Syndrome in Men and Women. The Journals of Gerontology: Series A. 2009;64A(1):69-75.

38.Pabst G, Zimmermann AK, Huth C, Koenig W, Ludwig T, Zierer A, et al. Association of low 25-hydroxyvitamin D levels with the frailty syndrome in an aged population: results from the KORA-age Augsburg study. The journal of nutrition, health \& aging. 2015;19(3):258-64.

39.Puts MTE, Visser M, Twisk JWR, Deeg DJH, Lips P. Endocrine and inflammatory markers as predictors of frailty. Clinical Endocrinology. 2005;63(4):403-11.

40.Chang C-I, Chan D-C, Kuo K-N, Hsiung CA, Chen C-Y. Vitamin D insufficiency and frailty syndrome in older adults living in a Northern Taiwan community. Arch Gerontol Geriatr. 2010;50:S17-S21.

41.Zeng Y, Vaupel JW. Chinese Longitudinal Healthy Longevity Survey (CLHLS), Biomarkers Datasets, 2009, 2012, 2014. Inter-university Consortium for Political and Social Research [distributor]; 2019.

42.Zeng Y, Vaupel J, Xiao Z, Liu Y, Zhang C. Chinese Longitudinal Healthy Longevity Survey (CLHLS), 1998-2014. Inter-university Consortium for Political and Social Research [distributor]; 2017.

43.Xiao Q, Wu M, Zeng T. Social support networks in Chinese older adults: health outcomes and health related behaviors: a path analysis. Aging \& mental health. 2019:1-9.

44.Zeng Y, Feng Q, Hesketh T, Christensen K, Vaupel JW. Survival, disabilities in activities of daily living, and physical and cognitive functioning among the oldest-old in China: a cohort study. Lancet. 2017;389(10079):1619-29.

45.Lv X, Li W, Ma Y, Chen H, Zeng Y, Yu X, et al. Cognitive decline and mortality among community-dwelling Chinese older people. 2019;17(1):63.

46.Liu Z, Han L, Feng Q, Dupre ME, Gu D, Allore HG, et al. Are China's oldest-old living longer with less disability? A longitudinal modeling analysis of birth cohorts born 10 years apart. BMC Med. 2019;17(1):23.

47.Lv YB, Gao X, Yin ZX, Chen HS, Luo JS, Brasher MS, et al. Revisiting the association of blood pressure with mortality in oldest old people in China: community based, longitudinal prospective study. Bmj. 2018;361:k2158.

48.Sun D, Sun X, Xu Y, Wu T, Tao L. Superoxide dismutase activity and risk of cognitive decline in older adults: Findings from the Chinese Longitudinal Healthy Longevity Survey. Experimental gerontology. 2019;118:72-7.

49.Yin ZX, Shi XM, Kraus VB, Fitzgerald SM, Qian HZ, Xu JW, et al. High normal plasma triglycerides are associated with preserved cognitive function in Chinese oldest-old. Age Ageing. 2012;41(5):600-6.

50.Dupre ME, Gu D, Warner DF, Yi Z. Frailty and type of death among older adults in China: prospective cohort study. Bmj. 2009;338:b1175.

51.Gaudry M, J. Wills M. Estimating the functional form of travel demand models. Transportation Research. 1978;12:257-89.

52.Cole T. Applied logistic regression. D. W. Hosmer and S. Lemeshow, Wiley, New York, 1989. No. of pages: xiii + 307. Price: £36.00. Statistics in Medicine - STAT MED. 1991;10:1162-3.

53.Turlach B. Spline Smoothing. 2016. p. 1-8.

54.B. Onukogu I. When is Piece-Wise Regression Really Necessary? Biometrical Journal - BIOM J. 1984;26:559-66.

55.Bahrami A, Sadeghnia HR, Tabatabaeizadeh SA, Bahrami-Taghanaki H, Behboodi N, Esmaeili H, et al. Genetic and epigenetic factors influencing vitamin D status. 2018;233(5):4033-43.

\section{Tables}


Table 1. Participant characteristics.

Variables All participants

Categories (nmol/L)

statistics

\begin{tabular}{lllll}
\cline { 3 - 4 }$(\mathrm{n}=1324)$ & $\mathrm{Q}_{1}(\leq 26.13)$ & $\mathrm{Q}_{2}(26.13-35.89)$ & $\mathrm{Q}_{3}(35.89-50.00)$ & $\mathrm{Q}_{4}(>50.00)$
\end{tabular}

\section{Socio-}

demographics, $n$

(\%)

Age (80-112), M 92.89(7.92)

95.63(7.49)

93.37(7.72)

91.85(7.85)

$90.70(7.78)$

$25.207^{* * *}$

(SD)

\begin{tabular}{lllllll}
\hline Female & $844(63.7)$ & $251(75.8)$ & $236(71.3)$ & $200(60.2)$ & $157(47.6)$ & $68.190^{* * *}$ \\
\hline Married & $294(22.3)$ & $44(13.3)$ & $60(18.2)$ & $83(25.1)$ & $107(32.7)$ & $40.503^{* * *}$ \\
\hline Rural & $1124(84.9)$ & $282(85.2)$ & $282(85.2)$ & $265(79.8)$ & $295(89.4)$ & $11.925^{* *}$ \\
\hline No schooling & $998(76.4)$ & $279(85.8)$ & $257(78.4)$ & $242(74.2)$ & $220(67.1)$ & $33.410^{* * *}$ \\
$\begin{array}{l}\text { With household } \\
\text { member(s) }\end{array}$ & $950(73.2)$ & $263(82.2)$ & $236(72.2)$ & $225(69.7)$ & $226(69.1)$ & $25.873^{* * *}$ \\
\hline
\end{tabular}

\section{Health}

characteristics,

n (\%)

\begin{tabular}{|c|c|c|c|c|c|c|}
\hline Smoking & $148(11.3)$ & $28(8.5)$ & $39(11.8)$ & $37(11.2)$ & $44(13.5)$ & 4.337 \\
\hline Drinking & $167(12.7)$ & $32(9.7)$ & $41(12.5)$ & $45(13.6)$ & $49(14.9)$ & 0.218 \\
\hline Regular exercise & 178(13.9) & $27(8.4)$ & $38(11.9)$ & $54(16.7)$ & $59(18.6)$ & $17.317^{* * *}$ \\
\hline Hypertension & $785(62.2)$ & 197(61.9) & 195(62.1) & 192(61.5) & $201(63.0)$ & 0.155 \\
\hline $\begin{array}{l}\text { Diabetes } \\
\text { mellitus }\end{array}$ & $98(7.4)$ & $28(8.5)$ & $25(7.6)$ & $24(7.3)$ & $21(6.4)$ & 1.057 \\
\hline Heart diseases & $91(7.0)$ & $24(7.4)$ & $24(7.4)$ & $27(8.4)$ & 16(4.9) & 3.398 \\
\hline $\begin{array}{l}\text { Cerebrovascular } \\
\text { diseases }\end{array}$ & $102(7.8)$ & $34(10.4)$ & $31(9.5)$ & $18(5.5)$ & $19(5.8)$ & $8.630^{*}$ \\
\hline $\begin{array}{l}\text { Respiratory } \\
\text { diseases }\end{array}$ & $116(8.9)$ & $29(9.0)$ & $32(9.8)$ & $23(7.0)$ & $32(9.8)$ & 2.080 \\
\hline
\end{tabular}

\section{Biomarkers, $\mathrm{M}$}

(IQR)

\begin{tabular}{lllllll} 
CRP $(\mathrm{mg} / \mathrm{L})$ & $1.01(0.41,2.93)$ & $1.12(0.38,3.35)$ & $0.93(0.43,3.05)$ & $0.96(0.41,2.54)$ & $1.09(0.39,2.75)$ & 1.491 \\
\hline ALB $(\mathrm{g} / \mathrm{L})$ & $39.10(35.90,42.40)$ & $37.90(35.30,41.40)$ & $38.60(35.48,42.12)$ & $39.70(36.70,42.93)$ & $39.90(37.20,42.80)$ & $29.923^{* * *}$ \\
\hline CHO $(\mathrm{mmol} / \mathrm{L})$ & $4.16(3.52,4.79)$ & $4.03(3.49,4.72)$ & $4.21(3.51,4.79)$ & $4.21(3.47,4.97)$ & $4.20(3.70,4.78)$ & 4.186 \\
\hline CREA $(\mathrm{mmol} / \mathrm{L})$ & $78(65,96)$ & $69(60,85)$ & $77(63,93)$ & $82(69,98)$ & $87(71,102)$ & $76.765^{* * *}$ \\
\hline HDLC $(\mathrm{mmol} / \mathrm{L})$ & $1.23(1.03,1.49)$ & $1.20(1.01,1.45)$ & $1.25(1.04,1.51)$ & $1.27(1.03,1.55)$ & $1.23(1.04,1.46)$ & 5.065 \\
\hline LDLC $(\mathrm{mmol} / \mathrm{L})$ & $2.45(1.94,3.02)$ & $2.40(1.92,2.97)$ & $2.42(1.89,3.05)$ & $2.41(1.86,3.08)$ & $2.54(2.04,3.00)$ & 5.147 \\
\hline TG $(\mathrm{mmol} / \mathrm{L})$ & $0.79(0.59,1.10)$ & $0.78(0.59,1.07)$ & $0.79(0.58,1.09)$ & $0.82(0.61,1.16)$ & $0.77(0.57,1.07)$ & 6.429 \\
\hline SOD $(\mathrm{IU} / \mathrm{mL})$ & $58.53(53.43,63.24)$ & $56.75(51.75,62.97)$ & $58.18(53.49,63.20)$ & $58.75(53.33,63.06)$ & $59.39(55.39,64.24)$ & $18.975^{* * *}$ \\
\hline MDA $(\mu \mathrm{mol} / \mathrm{L})$ & $4.71(3.73,5.79)$ & $4.81(3.93,5.89)$ & $4.87(3.88,5.91)$ & $4.84(3.82,5.83)$ & $4.33(3.25,5.55)$ & $27.303^{* * *}$ \\
\hline WBC $\left(10^{9} / \mathrm{L}\right)$ & $5.30(4.30,6.40)$ & $4.80(4.00,6.00)$ & $5.10(4.10,6.10)$ & $5.60(4.57,6.60)$ & $5.60(5.60,6.80)$ & $34.983^{* * *}$ \\
\hline HGB $(\mathrm{g} / \mathrm{L})$ & $118(106,131)$ & $121(110,133)$ & $120(107,132)$ & $116(105,129)$ & $117(105,131)$ & $11.618^{* * *}$
\end{tabular}

Page 10/12 
Frailty, n (\%) 426(33.2)

M (SD), mean (standard variance); M (IQR), median (interquartile range).

${ }^{\text {a }}$ Coefficient of ANOVA, Kruskal-Wallis test or $\chi^{2}$ test among categories of plasma 25(OH)D level.

${ }^{*}<0.05,{ }^{* *}<0.01,{ }^{* * *}<0.001$.

Abbreviations: CRP, C reactive protein; ALB, plasma albumin; CHO, total cholesterol; CREA, plasma creatine; HDLC, high-density lipoprotein cholesterol; LDLC, low-density lipoprotein cholesterol; SOD, superoxide dismutase; TG, triglyceride; SOD, superoxide dismutase; MDA, malondialdehyde; WBC, white blood cell count; HGB, hemoglobin.

\section{Figures}

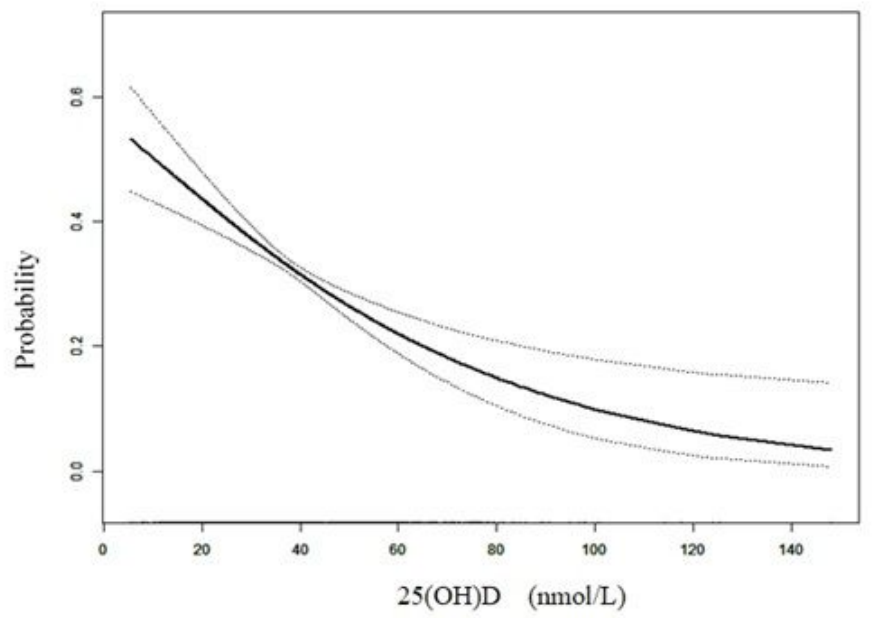

Figure 1. The dose-response relationship of $25(\mathrm{OH}) \mathrm{D}$ level and risk of frailty in the full adjusted model. Lines $=$ estimated probability of frailty with $25(\mathrm{OH}) \mathrm{D}$, dotted lines $=95 \%$ confidence intervals.

Figure 1

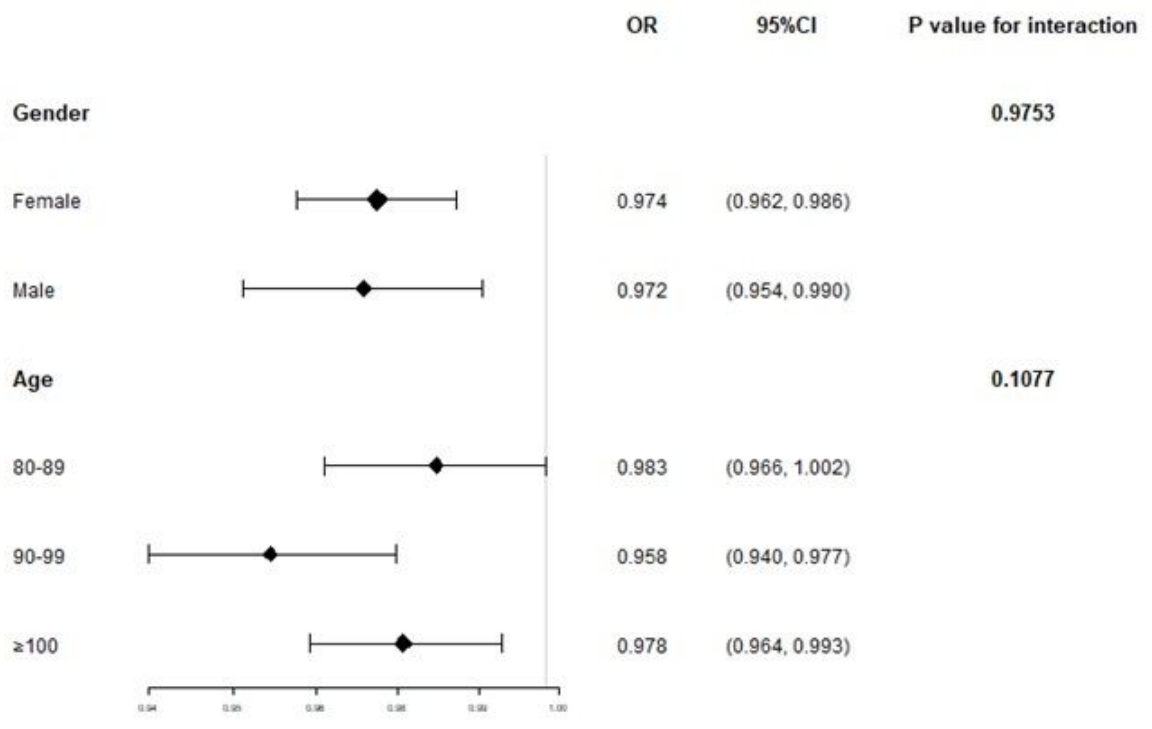

Figure 1. Subgroup analyses for the association between $25(\mathrm{OH}) \mathrm{D}$ and frailty in the fully adjusted model.

Figure 2 


\section{Supplementary Files}

This is a list of supplementary files associated with this preprint. Click to download.

- Additionalfile1SupplementaryTables.docx

- Additionalfile3STROBEchecklist.docx

- Additionalfile2Authorsinformation.docx 